\title{
CRENÇAS SOBRE PRÁTICAS: ESTUDO SOBRE MÃES PRIMÍPARAS DE CONTEXTO URBANO E NÃO-URBANO
}

\author{
BELIEFS ABOUT PRACTICES: STUDY ON MOTHERS \\ PRIMIPAROUS FROM URBAN AND NO-URBAN CONTEXT
}

Raimundo Arão Silva ${ }^{1}$

Celina Maria Colino Magalhães ${ }^{1}$

Silva RAM; Magalhães CMC. Crenças sobre práticas: um estudo sobre mães primíparas de contexto urbano e não-urbano. Rev. Bras. Cresc. e Desenv. Hum. 2011; 21(1): 39-50.

\section{Resumo:}

A literatura sobre cognições parentais tem indicado que mães de diferentes contextos e com diferenças no nível de escolaridade tendem a diferir quanto as suas crenças. O presente estudo teve como objetivo investigar as cognições parentais em mães de dois contextos diferentes. A amostra foi formada por 100 mães primíparas, sendo 50 de contexto urbano e 50 de contexto não-urbano. As participantes responderam ao Questionário de Crenças sobre práticas maternas. Os dados foram analisados de acordo com as categorias propostas pelos autores dos instrumentos. Os resultados indicaram que as mães dos dois contextos diferiram quanto a idade, escolaridade e valor dos escores na avaliação de crenças sobre práticas. $\mathrm{O}$ nível de escolaridade materna correlacionou-se positivamente com a idade, com os escores da avaliação das práticas. Foi verificado que as mães dos dois contextos assemelham-se quanto ao nível de importância atribuído a algumas práticas, mas diferenciam-se em relação a outras e que apresentam a mesma ordem de valorização das dimensões de crenças. Os resultados encontrados corroboram dados da literatura nacional que tem indicado que as mães brasileiras tendem a valorizar práticas relacionadas a estimulação. Estes resultados contribuem para a ampliação da compreensão das cognições de mães brasileiras e para fortalecer as evidencias dos efeitos do contexto e da escolaridade sobre as cognições parentais.

Palavras-chave: cognições parentais; crenças; metas e estratégias de socialização.

1 Universidade Federal do Pará, Belém, Brasil

Correspondência para: Raimundo Arão Silva. E-mail: raimundoarao@gmail.com 


\begin{abstract}
:
The literature about parental cognitions has been indicating that mothers from different contexts and with differences in the educational level tend to shows different beliefs. The present study has as objective investigates the parental cognitions in mothers from two different contexts. The sample was formed by 100 primiparous mothers, being 50 from urban context and 50 from no-urban context. The participants answered to the Questionnaire of beliefs about maternal practices. The data were analyzed in agreement with the categories proposed by the authors of the instrument. The results indicated that the mothers of the two contexts differed as the age, education and value of the scores in the evaluation of beliefs on maternal practices. The level of maternal education was correlated positively with the age, with the scores of the evaluation of the practices. It was verified that the mothers from two contexts resemble each other as for the level of importance attributed the some practices, but they differ in relation to other and that they present the same order of valorization of the dimensions of beliefs. The results found corroborate data of the national literature that it has been indicating that the Brazilian mothers tend to value practices related to stimulation. These results contribute to the enlargement of the understanding of the Brazilian mothers' cognitions and to strengthen the evidence of the effects of the context and of the education on the parental cognitions.
\end{abstract}

Key words: parental cognitions; beliefs; goals and socialization strategies.

\section{INTRODUÇÃO}

O modo como crianças são cuidadas variou significativamente ao longo da história da humanidade, evidenciando-se especificamente a história da civilização ocidental. Podemos inferir que continuam a ocorrer modificações no modo como os pais e cuidadores lidam com crianças de diferentes faixas etárias. As especificidades no trato com infantes são influenciadas tanto pelo contexto cultural quanto pelas características particulares do grupamento familiar.

Harkness e Super ${ }^{1}$ propuseram o conceito de Nicho de Desenvolvimento, composto por três elementos básicos: (a) o ambiente físico e social (micro e macro) onde a criança vive; (b) as práticas culturais de cuidado e educação de crianças, prescritas socialmente; e (c) as crenças e valores dos cuidadores das crianças, suas concepções de infância, desenvolvimento e educação, ditadas pela cultura do grupo ao qual pertencem.
As crenças e valores situam-se no campo das etnoteorias parentais, definidas como construções pessoais, coletivamente organizadas por indivíduos que assumem papéis parentais e por crenças culturais e ideologias comunicadas na forma de sugestões sociais².

Assumindo que o contexto sóciocultural se constitui em importante fonte de informações, que podem variar significativamente de um grupo cultural para outro e entre diferentes épocas na mesma sociedade, torna-se essencial a compreensão desse contexto como fonte de subsídios para formação de crenças e valores.

Muitos estudiosos têm se dedicado à investigação da Psicologia Parental. Miller ${ }^{3}$ e Goodnow $^{4}$ publicaram importantes revisões de literatura sobre esse tema. Nessas revisões notam-se ausência de consenso quanto à terminologia utilizada para se referir ao objeto de estudo.

Referindo-se a esta falta de consenso claramente explicitada por Sigel, et al. ${ }^{5}$ na segunda edição do "Parental Belief System: 
psychological consequences for children” Ribas $^{6}$, assume posição favorável ao uso do termo “cognições parentais" proposto pelos autores e destaca que crenças podem ser entendidas como os mais diversos tipos de proposições simples acerca da relação entre duas coisas ou entre uma coisa e uma de suas características.

Para Ribas" o termo "crenças" se relaciona com estereótipos e atitudes, sendo por essa razão um conceito central para a Psicologia, englobando ainda o conceito de valores, entendido modos de conduta e estados finais desejados ou indesejados (crenças prescritivas), contém também as metas de socialização, visto que as metas são definidas como representações internas de estados finais desejados.

No presente estudo os termos crenças e metas foram entendidos e utilizados como conceitos incluídos dentro do conceito mais amplo de cognições parentais no sentido descrito por Ribas ${ }^{6}$, como "um amplo conjunto de produtos (por exemplo: crenças, valores, estereótipos, expectativas) e processos psicológicos (por exemplo: atribuição de causalidade, autopercepção, julgamento), relacionados ao desenvolvimento humano, à maternidade e à paternidade".

O termo "crenças sobre práticas" foi utilizado para descrever as proposições ou interpretações das mães acerca da importância/adequação de determinadas práticas de cuidado com suas crianças.

É conveniente supor que o modo como uma criança inserida em um contexto urbano tecnológico se desenvolve difere do modo como se processa o desenvolvimento de uma criança da mesma idade em uma sociedade não urbana. A disponibilidade de informação, recursos tecnológicos e a possibilidade de aprendizado em diferentes áreas de conhecimento são fatores importantes na determinação do que pode ser feito em termos de investimento em cuidado e formação para as crianças, bem como na definição das expectativas que se tem acer- ca de seu desenvolvimento físico, social, intelectual e profissional.

Keller $^{7}$ apresenta duas trajetórias ontogenéticas que configuram dois diferentes modos de investimento parental, um modelo ocidental e urbano e outro não-ocidental, nãourbano. O padrão ocidental, essencialmente urbano, caracteriza-se por períodos mais curtos de amamentação (um a três meses), por períodos reduzidos em que o bebê é carregado no colo e reduzido contato físico, pois o bebê passa a maior parte do tempo em berços e/ou em carrinhos e geralmente recebe cuidados extras de pessoas pagas para esse fim (babás e creches). O cuidado emocional disponibilizado promove a independência precoce e envolve curtos períodos de interação face-a-face da criança com outras pessoas.

O padrão não-ocidental/não-urbano, por outro lado, é marcado por longos períodos de amamentação ( 2 a 4 anos) e o bebê é carregado a maior parte do tempo durante seus primeiros anos. O contato corporal se dá durante o dia e a noite (nas costas, frente ou quadril da mãe), as crianças dormem com os adultos e os cuidados extras são oferecidos por outros parentes e irmãos da criança. O cuidado emocional apóia uma longa simbiose com a mãe e se caracteriza por longos períodos de cuidado que ocorrem conjuntamente a outras atividades do adulto, há prolongado contato e comunicação corporal.

Para Keller ${ }^{8}$, essas diferenças entre as formas de criação entre os centros urbanos e ambientes rurais, ocasiona à valorização de dois padrões extremos, gerando tipos de psicologias extremamente diferentes, ou seja, a construção de dois tipos diferentes de self. Nos contextos não urbanos há uma tendência para desenvolvimento de um self mais voltado a construção de vínculos interpessoais estreitos como um aspecto de interdependência, enquanto em contextos urbanos encontra-se uma tendência para a construção de um self voltado para relações mais abertas (distantes/separadas) como um aspecto de independência. 
Fundamentada em um conjunto de dados provenientes de pesquisas empíricas Keller $^{8}$, propõe a existência de um componente de Modelo de Parentagem constituído de um repertório universal de sistemas de parentagem evoluídos filogeneticamente e que são moldados individualmente por mecanismos interacionais. Os sistemas de parentagem são: cuidado primário, contato corporal, estimulação corporal, estimulação por objetos, trocas face-a-face e envelope narrativo (narrative envelope), definidos com base em comportamentos específicos e são considerados como sistemas funcionalmente relacionados entre si.

Considerando esta associação dos sistemas de parentagem com contextos que estão ligados ao desenvolvimento de padrões de metas e self diferentes, pode-se inferir também uma relação mesmo que indireta entre os sistemas de parentagem e as crenças dos pais em relação aos filhos.

A literatura sobre cognições parentais cresce constantemente e como destacado por Krepnner ${ }^{9}$ tem aumentado o número de investigações da família como "nicho primário" de sobrevivência e socialização, a família é compreendida como transmissora de significado e cultura. Pesquisas sobre desenvolvimento infantil investigam as crenças dos pais e as relações existentes entre crenças e práticas de cuidado e educação, bem como as variáveis que influenciam as crenças dos pais em determinada direção.

Estudos têm destacado que a escolaridade 10-13, o contexto cultural e classe social 14-21 têm importantes implicações na construção das crenças, e consequentemente do modo de se comportar, dos adultos em relação às crianças.

Merece destaque o estudo conduzido por Suizzo ${ }^{22}$ sobre os modelos culturais e crenças de pais e mães parisienses sobre criação de filhos. Na investigação foi utilizado o Questionário de crenças e ideias sobre crianças e bebês (Croyances et Idéas sur les Nourrissons et
Petis Enfants - CINPE, ou Beliefs and Ideas about Iinfants and yong children), objetivando averiguar como crenças discretas sobre práticas de criação podem está inter-relacionadas no pensamento de pais parisienses.

Os resultados indicaram tendência dos participantes a valorizarem práticas relacionadas a: estimulação cognitiva, higiene básica, proximidade interpessoal e promoção da individualidade, estimulação do lactente por meio de exposição a objetos, pessoas e sabor de alimentos, bem como manutenção da higiene e socialização para interação pública.

Também foram identificadas três escalas ou modelos culturais de parentagem dos pais parisienses: a) "estimular a criança" pelo fato de estar associado a práticas dirigidas à exposição da criança a um número de diferentes estímulos, oportunidades para interações diádicas e grupais, com brinquedos, através da linguagem, bem como através do paladar e tato; b) “apresentação apropriada”, pois é composto por práticas associadas à garantia de que a criança aparecerá (será vista) bem comportada e limpa, tais como as práticas de higiene (banho diário, manter coisas sujas fora da boca da criança e trocar fraldas) e regras de socialização (comportar-se bem em público, cumprimentar, agradecer e ter controle emocional); e c) "responsividade e vínculo" contém itens associados ao atendimento das necessidades da criança em primeiro plano, em vez de impor a vontade do adulto sobre ela.

A autora identificou diferenças nas crenças em função de variáveis sociodemográficas, como nível educacional, idade, gênero e nível de aculturação.

Nos estudos relatados acima pode ser visualizada a influência de diversas variáveis, como contexto sóciocultural, escolaridade/conhecimento teórico, gênero, número de filhos e classe sócioeconômica, sobre as cognições parentais, mesmo que esse termo não tenha sido utilizado em todos os estudos citados. A literatura também tem sugerido que as cognições pa- 
rentais podem ter influências sobre a forma como os pais ou cuidadores lidam com as crianças, produzindo resultados diferentes no que se refere a quantidade e qualidade do desenvolvimento alcançado por cada indivíduo.

No Brasil as investigações sobre a temática podem ser consideradas como em fase de implementação, mas já dispõem de um volume razoável de trabalhos identificados como estudo das cognições parentais 23-31 e a exemplo das publicações estrangeiras têm indicado significativa influência das variáveis sociodemográficas sobre o modo como pais e cuidadores/educadores concebem e lidam com crianças

Apesar da existência de uma literatura nacional relacionada à área de cognições parentais, ainda há carência de estudos específicos sobre as cognições de mães brasileiras, que comparem as populações das diversas regiões e contextos culturais do Brasil destacando as diferenças e semelhanças nas crenças e metas dessas populações.

Nos trabalhos descritos acima nota-se que na literatura estrangeira alguns estudos 10,11 investigaram crenças fazendo referências aos contextos urbanos e rurais. Na literatura nacional destaca-se apenas o trabalho de Rue$\mathrm{la}^{27}$ que investigou crenças e metas entre mães e avós de contexto rural.

Assim, o presente estudo investiga as práticas educativas de mães primíparas no contexto urbano e não-urbano.

\section{MÉTODO}

\section{Participantes}

Fizeram parte do estudo cem mães primíparas com no mínimo 18 anos de idade $(\mathrm{M}=23,82 / \mathrm{SD}=5,13)$ com filho com idade de zero a 59 meses $(M=17,62 / S D=12,71)$, sendo 50 de contexto urbano e 50 de contexto não-urbano. A decisão de estudar esta faixa etária deveu-se ao fato de que o instrumento que foi utilizado envolvia questões somente aplicáveis a crianças pequenas. A escolha de investigar as crenças em mães primíparas levou em consideração que esta é uma área de pesquisa que já vem sendo desenvolvida no Brasil, por Seidl de Moura et al ${ }^{32}$ em áreas urbanas, e que amostras não-urbanas precisam ser investigadas.

Os dados foram coletados em dois contextos distintos, sendo um urbano na cidade de Belém e outro não-urbano nos povoados do município de Santa Bárbara do Pará, para os quais foram consideradas a definições da Lei Municipal n 091 de 05 de outubro de 2006 que estabelece o Plano Diretor do Município de Santa Bárbara do Pará e concebe a ordenação espacial do município em três níveis: áreas urbanas, áreas de expansão urbana e áreas rurais.

\section{Instrumento}

A fim de obter as informações necessárias à consecução dos objetivos propostos, foi utilizado o Questionário de Crenças sobre Práticas Maternas, criado por Suizzo (22) e adaptado e validado por Seidl de Moura (32), instrumento composto por 50 itens, sendo 25 relacionados a lactentes até um ano e 25 para crianças entre um e três anos. O questionário contém, ainda, perguntas que levantavam dados sociodemográficos do filho (idade e sexo) e dos seus pais (idade, instrução e ocupação).

\section{Procedimentos}

O projeto de pesquisa foi submetido ao Comitê de Ética em Pesquisa (CEP) do Núcleo de Medicina Tropical de Universidade Federal do Pará e recebeu a aprovação sob o protocolo número 014/2007 - CEP/NMT. De posse da autorização iniciou-se o treinamento de uma equipe de quatro discentes do curso de graduação em Psicologia a fim de habilitá-los para aplicação precisa dos instrumentos utilizados no estudo. 
Na aplicação do Questionário sobre Crenças e Práticas o pesquisador explicou as participantes que iria ler 50 afirmações e que ela deveria atribuir nota de "zero" a "cinco" para cada uma delas, de acordo com o seu nível de concordância, onde zero significava 'não concordo', um significava 'não é importante', dois 'é pouco importante', três é razoavelmente importante', quatro'é muito importante' e cinco 'é extremamente importante'. Passando a seguir para a leitura da primeira assertiva: "Estimular a criança a brincar com outras de costumes diferentes", e citando cada nota e seu significado, a seguir solicitava à mãe que atribuísse a sua nota a esta afirmação e aguardava a sua resposta para registrá-la no campo apropriado.

No tratamento dos dados sóciodemográficos optou-se por utilizar a idade das mães e das crianças em seus valores absolutos para o procedimento de análise, para verificar com mais precisão o efeito destas variáveis sobre as crenças acerca das práticas. O sexo das crianças foi agrupado em masculino e feminino. $\mathrm{O}$ nível educacional das mães foi classificado em uma escala de sete níveis: de 01 - ensino fundamental incompleto a 07 - pós-graduação.

Os dados foram lançados em uma planilha do SPSS a fim de se proceder a aplicação do teste $t$ de Student para verificar a existência de diferenças significativas entre as duas amostras e cruzar estas variáveis com os dados provenientes do Questionário de Crenças sobre Práticas.

Os dados provenientes do Questionário Crenças sobre Práticas Maternas - Croyances et idées sur lês nourissons et petits enfants CINPE 22 foram lançados no pacote estatístico SPSS e para possibilitar as análises foram necessárias primeiramente a limpeza e organização dos dados na planilha.

Considerando o fato de que o número total de participantes do estudo $(\mathrm{N}=100)$ foi insuficiente para a realização de uma análise fatorial, utilizou-se o agrupamento de assertivas provenientes da análise fatorial realizada com uma amostra de mães de sete capitais brasileiras, feita pelo grupo de estudos nacional sobre crenças 31 .

Calculou-se escore médio de cada participante nas dimensões constantes do inventário de Suizzo 22, as quais se relacionam com aspectos do desenvolvimento infantil, a saber: 1) Estimulação - chamar a atenção e expor a criança a diferentes estímulos (cognitivo e motor); 2) Apresentação - garantir a apresentação apropriada da criança (social); 3) Responsividade e vínculo - responder ao filho e criar vínculo com ele (emocional). 4) Disciplina - educação dos filhos no sentido maior de ensinar regras.

Para identificar diferenças significativas entre os escores médios das participantes dos dois contextos foi aplicado o Teste $t$ de Student para amostras independentes. Foram aplicados testes para verificar a existência de correlações ( $r$ ) entre as dimensões "estimulação" e "apresentação” e os fatores sóciodemográficos.

\section{RESULTADOS E DISCUSSÃO}

Seguindo indícios da literatura que sugerem que as variáveis sociodemográficas têm influências sobre as cognições parentais, adotou-se a idade e a escolaridade materna, bem como a idade e o sexo da criança como descritores das características das participantes do estudo. A seguir são apresentas os dados referentes a essas variáveis.

A média de idade da amostra total foi $\mathrm{M}=$ 23,82 ( $\mathrm{DP}=5,13$ ). As mães de Belém apresentaram média de idade maior que a das mães de Santa Bárbara, $\mathrm{M}=25,72(\mathrm{DP}=5,32) \mathrm{e}$ $\mathrm{M}=21,92(\mathrm{DP}=4,20)$ respectivamente e os resultados do teste $t$ de Student $\left(t_{(98)}=3,97\right.$, $p<0,001)$ revelou significativa diferença entre a média de idade das mães dos dois contextos.

A média de idade das crianças amostra total foi $\mathrm{M}=17,62$ ( $\mathrm{DP}=12,71)$, sendo que as de Belém tinham em média 15,86 meses (DP $=11,34)$, enquanto entre as de Santa Bár- 
bara tinham 19,38 (DP = 13,84). O resultado do teste $t$ de Student para amostras independentes $t_{(98)}=-1,39, p>0,05$, indicou que a diferença não possui significância.

A média de escolaridade das mães da amostra total foi $M=4,32$ (DP = 1,47), sendo das mães de Belém $M=2,12(\mathrm{DP}=0,63)$ enquanto que a média das mães de Santa Bárbara foi $M=1,64(D P=0,53)$. Neste caso o teste $t_{(98)}=4,148, p<0,001$ sugere que as mães de Belém possuem escolaridade significativamente maior que a das mães de Santa Bárbara. Em relação ao sexo das crianças, coincidentemente em ambos os contextos houve o mesmo número de participantes de cada sexo, sendo 22 crianças de sexo feminino e 28 de sexo masculino em cada.

As duas amostras se assemelham no tocante à idade e ao sexo das crianças, pois nestas duas variáveis foram encontradas diferenças não significativas entre as participantes dos dois contextos, entretanto, diferem quanto as variáveis maternas, pois os resultados do teste $t$ indicam que as mães de Belém apresentam idade e nível de escolarização significativamente maior que a idade das mães de Santa Bárbara.

Destaca-se ainda o fato de que a idade e a escolaridade materna se correlacionam positiva, $r=0,53, p<0,001$. A literatura tem relatado que a escolaridade materna possui importante papel preditor de crenças sobre desenvolvimento 32.

Considerando esses dados pode-se supor que as diferenças na expressão das crenças das dos dois contextos podem se dever aos efeitos das variáveis maternas, que, por sua vez, estão relacionadas a elementos do contexto sóciocultural mais amplo no qual estão inseridas.

A seguir são apresentados dados referentes aos modelos culturais ou dimensões de crenças maternas, as correlações entre as dimensões das crenças e destas com as variáveis sociodemográficas.

Os dados do Questionário de Crenças sobre Práticas foram analisados considerando as dimensões propostas por Suizzo ${ }^{22}$. Os resultados do teste $t$ comparando os escores médios das mães dos dois contextos em cada uma das dimensões com as respectivas médias e desvios padrões estão apresentados na tabela 1 a seguir.

Observa-se na tabela, que as dimensões "estimulação" e "apresentação apropriada do bebê em público" obtiveram os maiores valores tanto em Belém quanto em Santa Bárbara. Em ambos os casos os valores de $t_{(98)}=3,87$, $p<0,001$ e $t_{(98)}=5,39, p<0,001$, respectivamente indicam os escores das mães de Belém são significativamente maiores que os das mães de Santa Bárbara.

Na dimensão "responsividade e vínculo”, as mães de Santa Bárbara apresentaram escores mais altos $(\mathrm{M}=2,83$ e $\mathrm{DP}=0,69)$ que as de Belém, $(\mathrm{M}=2,30$ e $\mathrm{DP}=0,76)$ com $t_{(98)}=-3,64, p<0,001$ indicando elevado nível de significância. Na quarta e última dimensão não foram encontradas diferenças significativas entre os escores das participantes dos dois contextos.

Tabela 1: Médias, desvios padrão e resultados do teste $t$ para as dimensões de crenças

\begin{tabular}{|c|c|c|c|c|c|}
\hline \multirow[t]{2}{*}{ Fatores } & \multicolumn{2}{|c|}{ Belém } & \multicolumn{2}{|c|}{ Sta. Bárbara } & \multirow[t]{2}{*}{$\mathbf{T}$} \\
\hline & $\mathrm{M}$ & $\mathrm{DP}$ & $\mathrm{M}$ & $\mathrm{DP}$ & \\
\hline Estimulação & 4,12 & 0,41 & 3,64 & 0,48 & $5,39 * * *$ \\
\hline Apresentação apropriada do bebê em público & 4,25 & 0,47 & 3,86 & 0,63 & $3,87 * * *$ \\
\hline Responsividade e vínculo & 2,30 & 0,76 & 2,83 & $0,69-$ & $-3,64 * * *$ \\
\hline Disciplina & 2,14 & 0,76 & 2,04 & 0,95 & 0,678 \\
\hline
\end{tabular}

$\mathrm{n}=50, \mathrm{gl}=98, * * * p<0,001$ 
Nota-se que as mães dos dois contextos valorizaram as dimensões na mesma ordem (1) "Apresentação apropriada do bebê em público" (2) "Estimulação" (3) "Responsividade e vínculo" e (4) "Disciplina”.

Houve diferenças significativas entre as mães dos dois contextos nos escores de três das quatro dimensões, sendo que nas dimensões "estimulação" e "apresentação" as mães de Belém obtiveram médias maiores que as obtidas pelas mães de Santa Bárbara, enquanto na dimensão "responsividade e vínculo" as mães de Santa Bárbara apresentaram maior escore médio.

Observa-se portanto, que as crenças das mães dos dois contextos diferenciam-se em alguns aspectos, mas assemelham-se em outros. As diferenças referem-se ao fato de que as mães de Belém em geral atribuíram alto nível de importância para as práticas agrupadas em torno das dimensões "apresentação" e "estimulação”, ao passo que as mães de Santa Bárbara atribuíram nível de importância apenas mediano para as mesmas práticas.

As semelhanças, por outro lado, relacionam-se ao fato de que nas práticas agrupadas em torno das dimensões "responsividade e vínculo" e "disciplina” tanto as mães de Belém quanto as de Santa Bárbara atribuíram baixa nível de importância.

O fato de Santa Bárbara e Belém serem cidades localizadas na mesma região, e muito próximas geograficamente, torna compreensiva a tendência das mães de ambas as cidades a valorizarem práticas semelhantes. Esses resultados podem conter indícios de que alguns aspectos exercem diferentes níveis de influência sobre as crenças das amostras estudadas, ou seja, existem elementos culturais/contextuais amplos influenciando de maneira diferenciada as cognições das mães dos dois contextos.

Com base na literatura é possível relacionar essas diferenças principalmente à escolaridade materna, visto que as duas amostras se diferenciam quanto ao nível de instrução e que a escolaridade tem sido apontada como importante preditor de crenças maternas.

A valorização da dimensão "apresentação" em primeiro lugar e da dimensão "estimulação" em segundo indica que as mães de ambas as amostras divergem das participantes do estudo de Suizzo ${ }^{22}$, que valorizam em primeiro lugar as práticas contidas na dimensão “estimulação" e em segundo as práticas da dimensão "apresentação”.

Comparando os dados do presente estudo com os resultados encontrados por Ruela ${ }^{27}$, verifica-se que há semelhanças das mães de Belém e Santa Bárbara com mães e avós da cidade de Ribeirão de São Joaquim, no que se refere a atribuição de maior importância as práticas relacionadas com a "Apresentação apropriada do bebê em público”, seguidas das práticas ligadas a "Estimulação", que teve nível de importância mediana entre as participantes dos dois estudos.

No que se refere à valorização das dimensões "responsividade e vinculo" e "disciplina" em terceiro e quarto lugares, respectivamente, os dados aqui apresentados estão de acordo com os relatos da literatura nacional e internacional, pois em ambos os estudos citados estas duas dimensões obtiveram escores médios e apareceram na mesma ordem, sendo consideradas pouco importantes, exceto pelas avós do estudo de Ruela ${ }^{27}$ que atribuíram importância mediana às práticas destas dimensões.

No tocante às correlações entre as dimensões, no presente estudo "apresentação" correlacionou-se positiva e significativamente com "estimulação" ( $\mathrm{r}=0,527, p<0,001)$. A dimensão "estimulação" correlacionou-se positivamente a idade $(r=0,39, p<0,01)$ e escolaridade maternas $(r=0,36 p<0,01)$. Esses resultados sugerem que quanto maior a idade e a escolaridade das mães mais elas tendem a valorizar práticas relacionadas com a estimulação da criança.

“Apresentação” não teve correlação significativa nem com a idade nem com a escola- 
ridade das mães. Nenhuma das duas dimensões correlacionou-se com a idade das crianças. A dimensão "responsividade e vínculo" não se correlacionou nem com as demais dimensões nem com as variáveis sociodemográficas.

Os dados referentes à correlação entre as três dimensões de crenças e destas com as variáveis sóciodemográficas estão apresentados na tabela 2.
Outro aspecto que merece atenção refere-se à dimensão "Responsividade e vínculo" que foi a terceira mais valorizada entre as mães dos dois contextos, e nesse caso as mães de Santa Bárbara apresentaram escore médio significativamente maior que o das mães de Belém. No estudo de Ruela ${ }^{27}$ esta dimensão também foi a terceira mais valorizada, e as avós atribuíram significativamente mais importân-

Tabela 2: Correlação entre as dimensões Estimulação e Apresentação e as variáveis sóciodemográficas das mães dos dois contextos

\begin{tabular}{lrrrrrr}
\hline & $\mathbf{1}$ & $\mathbf{2}$ & $\mathbf{3}$ & $\mathbf{4}$ & $\mathbf{5}$ & $\mathbf{6}$ \\
\hline 1. Idade da Mãe & 1,00 & & & & & \\
2. Escolaridade da Mãe & $0,53^{* *}$ & 1,00 & & & & \\
3. Idade da Criança & 0,16 &,- 07 & 1,00 & & & \\
4. Estimulação & $0,39^{* *}$ & $0,36^{* *}$ & $-0,01$ & 1,00 & & \\
5. Apresentação & 0,19 & 0,10 & $-0,02$ &, $053^{* *}$ & 1,00 & \\
6. Responsividade e vínculo &,- 050 & $-0,14$ & $-0,07$ & 0,10 & 0,11 & 1,00 \\
\hline
\end{tabular}

* $\mathrm{p}<0.05^{* *} \mathrm{p}<0.01$.

cia às práticas relacionadas a está dimensão do que as mães.

Considerando que se trata de uma população com características urbanas (Belém) e de outra considerada como não-urbana (Santa Bárbara), é possível relacionar a diferença na valorização das práticas voltados ao atendimento das demandas e a formação de vínculos estreitos com os efeitos do "modelo ecocultural de desenvolvimento infantil" (Keller) que tende a se destacar em cada contexto, ou seja, em contexto não urbano há uma tendência para desenvolvimento de um self mais voltado a construção de vínculos interpessoais estreitos como um aspecto de interdependência, enquanto em contextos urbanos, por outro lado, encontra-se uma tendência para a construção de um self voltado para relações mais abertas (distantes/separadas) como um aspecto de independência.

Pode-se considerar, portanto, que as mães de Santa Bárbara, por estarem inseridas em um contexto onde predominam características não urbanas estariam apresentando uma tendência à valorização de self mais voltado a construção de vínculos interpessoais estreitos. As mães de Belém, por outro lado, por residirem em contexto com características urbanas estariam tendendo a construção de um self voltado para relações mais abertas ou independentes. Em relação à semelhança das mães de Santa Bárbara com a avós de Ribeirão de São Joaquim, referente aos maiores escores, uma possível explicação pode ser provável influência que as mães de Santa Bárbara recebem das avós de seus filhos, pois foi verificado, assistematicamente, que na maioria dos casos as participantes recrutadas em Santa Bárbara moram na mesma casa de sua mãe ou sogra ou em uma casa bem ao lado.

Mesmo considerando a importância dos resultados obtidos, os autores reconhecem a existência de limitações à generalização relacionadas ao tamanho da amostra, bem como 
a utilização de um instrumento adaptado, que foi desenvolvido para medir as crenças de mães européias. As cognições parentais acerca das práticas foram investigadas utilizando um inventário composto por 50 assertivas e análise foi executada em conformidade com modelo utilizado pela autora do referido instrumento. Entretanto considera-se que novos instrumentos, dirigidos a população brasileira, poderão ser desenvolvidos para uso em estudos futuros, com amostras maiores de mães

\section{REFERÊNCIAS}

1. Harkness, S. \& Super, C. M. The Developmental Niche: a conceptualization at the interface of cjild and culture. International Journal of Development, 1999; 9: 545569.

2. Bandeira TTA; Moura MLS; Vieira ML. Metas de socialização de pais e mães para seus filhos. Rev Bras Crescimento Desenvolv Humano. 2009; 19(3): 445-456.

3. Miller, S. A. Parent's beliefs about children's cognitive development. Child Development. 1988; 59: 259-285.

4. Goodnow, J. J. Parents' idéas, actions, and feedings: models and methods from developmental and social psychology. Child Development. 1988; 59: 286-320.

5. Sigel, I. E; McGillicudd-DeLisi, A. V. \& Harwood, J. J. Parental Belief Systems: the psycholgiacal consequences for children (2nd. ed.). Hilldale: Lawrence Erlbaum Associates: 1992.

6. Ribas, R. C. Cognições de mães brasileiras acerca da paternidade e do desenvolvimento humano: Uma contribuição ao estudo da psicologia parental. Projeto de Tese de Doutorado. Programa de Pós-Graduação em Psicologia Social. Universidade do Estado do Rio de Janeiro: 2002.

7. Keller, H. Diferentes caminhos de socialização até a adolescência. Revista Brasileira de Crescimento e desenvolvimento Humano. 1998; 8: 1-14.

8. Keller, H. Culture of Infancy. Mahwah, New Jersey. London. Lawrence Erlbaum Associates Publishers: 2007. e/ou pais, das várias regiões do Brasil. Assim, as cognições e os modelos culturais de parentagem de mães brasileiras poderão ser melhor compreendidos, levanto em conta as especificidades de cada contexto de desenvolvimento, possibilitando comparações intra e transculturais

9. Krepnner, K. \& Planck, M. The Child and The Family: Interdependence in developmental Pathways. Teoria e Pesquisa 2000: 16: 1-25.

10. Palácios, J. e Moreno, M. C. Parentnt' and adolescent's ideas on children: origins and transmission of intracultural diversity. In: S. Harkness \& C.M. Super (orgs.). Parents' cultural belief systems. (pp. 254-269). New York: The Guiford Press; 1996.

11. Martin, C. A. \& Johnson, J. E. Children's self-perceptions and mother's beliefs about development and competencies. In: I. E. Siegal; A. V. McGillicuddy-DeLise \& J. J. Goodnow (org.). Parental belief systems (pp. 95-113). Hillsdalex Lawrence Erlbaum, 1992.

12. Edwards, C. P.; Gandini, L. e Giovaninni, D. The contrasting developmental timetables of parents and preschool teachers in two cultural communities. In: S. Harkness \& C.M. Super (orgs.). Parents' cultural belief systems. (pp. 270-288). New York: The Guiford Press; 1996

13. Le Vine, A. R.; Miller, P. M.; Richman, A. L. \& Le Vine, S. Education and Motherinfant interaction: A Mexican Case Study. In: S. Harkness \& C.M. Super (orgs.). Parents' cultural belief systems. (pp. 254269). New York: The Guiford Press; 1996.

14. Harwood, R.; Schoelmerich, A.; VenturaCook, E. Schulze, P. A. \& Wilson, S. P. Culture and Class Influence on Anglo and Porto Rican Mothers' Beliefs Regarding Long-Term Socialization Goals and Chuild Behavior. 1996; 67: 2446-2461. 
15. Harwood, R. L.; Schoelmeirch, A.; Schulze, P. A. e Gonzalez, Z. Cultural differences in Maternal Beliefs e Behaviors: A study of Middle-Class Anglo Puerto Rican Mother-Infant Pars in four everyday situations. Child Development, 1999; 70(4): 1005-1016.

16. Tudge, J.; Hogan, D.; Lee, S.; Tammeveski, M.M.; Kulakova, I. \& Putnam, S. Cultural heterogeneity: Parental values and beliefs and their preschoolers' activities in the United States, South Korean, Russia and Estonia. In A. Goneu (org.), Children's engagement in the world: Sociocultural perspectives. New York. Cambridge University Press. 1999. pp 62-96.

17. Baker, L.; Mackler, K.; sonnenschein, S. \& Serpell, R. Parents' interation with their First-Grade Children during Storybook readung and relations with subsequent home activity and Reading achievement. Journal of School Psychology. 2001; vol. 39, n.5: 415-438.

18. Vinden, P. G. Parenting atitudes and children's understanding of mind: A comparison of Korean American and AngloAmerican families. Cognitive Development. 2001; 16: 793-809.

19. Miller, A. M. \& Harwood, R. L. Long-term socialization goals and the construction of infants' network among middle class Anglo and Puerto-Rican mothers. International Journal of Behavioral Development, 2001; 23: 450-457.

20. Leyerdecker, B. Lamb, M. E. Harwood, R. L. \& Sholmerich, A. Mother's socialization goals and evaluation of deseirable and undesirable everyday situations in two diverse cultural groups. International Journal of Behavioral Development. 2002; 26: 248-258.

21. Citlak, B.; Leyerdecker, B.; Schöelmerich, A.; Driessen, R. \& Horwood, R. (2008). Socialization goals among first- and second-generation migrant Turkish and German mothers. International Journal of Behavioral Development. 2008; 32-56.

22. Suizzo, M. A. French Parents' cultural models and childrearing beliefs. International Journal of Behavioral Develoment. 2002; 26, 297-37.
23. Melo, C. (1996). Crenças maternas sobre desenvolvimento e educação de crianças em contexto de baixa renda. Dissertação de mestrado. Universidade de Brasília.

24. Lordelo, E.; Fonseca, A. e Araújo, M. L.V.B. Responsividade do ambiente de desenvolvimento: crenças e práticas como sistema cultural de criação de filhos. Psicologia: Reflexão e Crítica. 2000. 13; 73-80.

25. Seidl, M. L. M.; Bastos, A. C.S.; Ribas, R.C.R.; Vieira, M.; Piccinini, C. A. e Magalhães, C. M. C. Nicho de desenvolvimento de crianças brasileiras. conhecimento sobre desenvolvimento infantil em mães primiparas: influências dos fatores, diferenças regionais, nível socioeconômico, idade, escolaridade materna, idade e sexo do bebê. In: III Congresso Norte-Nordeste de Psicologia. João Pessoa. (2003)

26. Seidl de Moura, M. L.; Ribas Jr., R. C.; Piccinini, C. A.; Bastos, A. C. S.; Magalhães, C. M. C.; Vieira, M. L.; Salomão, N. M. R.; Silva, A. M. P. M. e Silva, A. K. Conhecimento sobre desenvolvimento infantil de mães primiparas de diferentes centros urbanos do Brasil. Estudos de Psicologia. 2004. 9(3). pp. 421-429.

27. Ruela, S. F. (2006) Um Estudo Intergeracional de Crenças Valorizadas por Mães em uma Comunidade Rural do Estado do Rio de Janeiro. [Dissertação]. Programa de Pós-Graduação em Psicologia Social. Universidade do Estado do Rio de Janeiro. Rio de Janeiro. 2006.

28. Vieira, M. L.; Seidl de Moura, M. L.; Lordelo, E. R.; Piccinini, C.; Magalhães, C. M. C.; Pontes, F.A.P. et al. Concepções de mães de sete capitais brasileiras sobre as metas de socialização de seus filhos: analise lexical através do programa informático ALCESTE. Anais do V Congresso Norte Nordeste de Psicologia. 2007. p. 746. [Acesso em 13/01/2008] disponível em http://www.conpsi5.ufba.br

29. Lordelo, E.; Seidl de Moura, M. L.; Vieira, M.; Piccinini, C. A.; Magalhães, C. M. C.; Pontes, F. A. R. e Mochizuki. Metas de Socialização em sete capitais brasileiras: Análise da ordem de evocação e frequência de descritores. Anais do V Congresso Norte Nordeste de Psicologia. 2007. 
p. 746. [Acesso em 13/01/2008] disponível em http://www.conpsi5.ufba.br

30. Seidl de Moura, M. L.; Vieira, M. L.; Lordelo, E.R.; Piccinini, C.;Magalhães, C. M. C. M. e Pontes, F. A. R. (2007). Metas de socialização de mães de sete cidades brasileiras: uma analise de conteúdo. Anais do V Congresso Norte Nordeste de Psicologia. 2007. p. 746. [Acesso em 13/01/ 2008] disponível em http:// www.conpsi5.ufba.br

31. Vieira, M. L.; Seidl de Moura, M. L.; Martins, G. D. F., Macarini, S. M., Rimo- li, A., Magalhães, C. M. C.; Pontes, F.A.P. et al (s/d). Beliefs about pratices in a group of Brazilan mother Running head: Parebtal pratices in Brazilan mother. (manuscrito da primeira versão do artigo em preparação para ser submetido).

32. Seidl de Moura, Maria Lucia; Bastos, Ana Cecília; Picinini, Cesar; Vieira, Mauro; Salomao. Brazilian mothers socialization goals: Intracultural differences in seven cities. International Journal of Behavioral Development.

Recebido em:17/abr./10

Modificado em: 10/jul./10

Aceito em: 28/dez./10 